\section{Sleep Apnea Syndrome}

Dona Locke

Psychiatry and Psychology, Mayo Clinic,

Scottsdale, AZ, USA

\section{Definition}

Central sleep apnea syndromes are characterized by a cessation or decrease of airflow due to absent or reduced respiratory effort. This may co-occur with obstructive sleep apnea (OSA). Diagnostic criteria by the ICSD-3 lists eight syndromes

1. Central sleep apnea with Cheyne-Stokes breathing

2. Central sleep apnea due to a medical disorder without Cheyne-Stokes breathing
3. Central sleep apnea due to high altitude periodic breathing

4. Central sleep apnea due to a medication or substance use

5. Primary central sleep apnea

6. Primary central sleep apnea of infancy

7. Primary central sleep apnea of prematurity

8. Treatment-emergent central sleep apnea

\section{Cross-References}

Obstructive Sleep Apnea Syndrome

\section{References and Readings}

American Academy of Sleep Medicine. (2014). International classification of sleep disorders (3rd ed.). Chicago: American Academy of Sleep Medicine. 